\title{
The Faber polynomial expansion method and its application to the general coefficient problem for some subclasses of bi-univalent functions associated with a certain $q$-integral operator
}

\author{
Hari Mohan Srivastava, Shahid Khan, Qazi Zahoor Ahmad, Nazar
} Khan and Saqib Hussain

\begin{abstract}
In our present investigation, we first introduce several new subclasses of analytic and bi-univalent functions by using a certain $q$-integral operator in the open unit disk $\mathbb{U}=\{z: z \in \mathbb{C}$ and $|z|<1\}$. By applying the Faber polynomial expansion method as well as the $q$-analysis, we then determine bounds for the $n$th coefficient in the Taylor-Maclaurin series expansion for functions in each of these newly-defined analytic and bi-univalent function classes subject to a gap series condition. We also highlight some known consequences of our main results.
\end{abstract}

Mathematics Subject Classification (2010): 05A30, 30C45, 11B65, $47 \mathrm{~B} 38$.

Keywords: Analytic functions, univalent functions, Taylor-Maclaurin series representation, Faber polynomials, bi-inivalent functions, $q$-derivative operator, $q$ hypergeometric functions, $q$-integral operators.

\section{Introduction and definitions}

Let $\mathcal{A}$ be the class of all functions $f$ which are analytic in the open unit disk

$$
\mathbb{U}=\{z: z \in \mathbb{C} \text { and }|z|<1\}
$$

and normalized by

$$
f(0)=0=f^{\prime}(0)-1 \text {. }
$$

Thus, clearly, the function $f \in \mathcal{A}$ has the following Taylor-Maclaurin series representation:

$$
f(z)=z+\sum_{n=2}^{\infty} a_{n} z^{n} \quad(z \in \mathbb{U}) .
$$

Further, by $\mathcal{S} \subset \mathcal{A}$ we shall denote the class of all functions which are univalent in $\mathbb{U}$. 
For two functions $f, g \in \mathcal{A}$, the function $f$ is said to be subordinate to the function $g$ in $\mathbb{U}$, denoted by

if there exists a function

$$
f(z) \prec g(z) \quad(z \in \mathbb{U})
$$

$$
w \in \mathbb{B}_{0}:=\{w: w \in \mathcal{A}, \quad w(0)=0 \quad \text { and } \quad|w(z)|<1 \quad(z \in \mathbb{U})\}
$$

such that

$$
f(z)=g(w(z)) \quad(z \in \mathbb{U}) .
$$

In the case when the function $g$ is univalent in $\mathbb{U}$, we have the following equivalence:

$$
f(z) \prec g(z) \quad(z \in \mathbb{U}) \Longleftrightarrow f(0)=g(0) \quad \text { and } \quad f(\mathbb{U}) \subset g(\mathbb{U}) .
$$

Next, for a function $f \in \mathcal{A}$ given by (1.1) and another function $g \in \mathcal{A}$ given by

$$
g(z)=z+\sum_{n=2}^{\infty} b_{n} z^{n} \quad(z \in \mathbb{U}),
$$

the convolution (or the Hadamard product) of the functions $f$ and $g$ is defined by

$$
(f * g)(z)=z+\sum_{n=2}^{\infty} a_{n} b_{n} z^{n}=(g * f)(z) .
$$

It is well known that every univalent function $f$ has an inverse $f^{-1}$, defined by

$$
f^{-1}(f(z))=z=f\left(f^{-1}(z)\right) \quad(z \in \mathbb{U})
$$

and

$$
f\left(f^{-1}(w)\right)=w \quad\left(|w|<r_{0}(f) ; r_{0}(f) \geqq \frac{1}{4}\right)
$$

where

$$
f^{-1}(w)=w-a_{2} w^{2}+\left(2 a_{2}^{2}-a_{3}\right) w^{3}-\left(5 a_{2}^{3}-5 a_{2} a_{3}+a_{4}\right) w^{4}+\cdots .
$$

A function $f \in \mathcal{A}$ is said to be bi-univalent in $\mathbb{U}$ if both $f$ and $f^{-1}$ are univalent in $\mathbb{U}$. We denote the class of all such functions by $\Sigma$. In recent years, the pioneering work of Srivastava et al. [22] essentially revived the investigation of various subclasses of the analytic and bi-univalent function class $\Sigma$. In fact, in a remarkably large number of sequels to the pioneering work of Srivastava et al. [22], several different subclasses of the analytic and bi-univalent function class $\Sigma$ were introduced and studied analogously by the many authors (see, for example, [5], [7], [9], [23], [24], [25], [28] and [29]). However, only non-sharp estimates on the initial coefficients $\left|a_{2}\right|$ and $\left|a_{3}\right|$ in the TaylorMaclaurin series expansion (1.1) were obtained in these recent papers.

The Faber polynomials introduced by Faber [11] play an important rôle in various areas of mathematical sciences, especially in Geometric Function Theory of Complex Analysis (see, for details, [27]). Recently, several authors (see, for example, [13] and [26]; see also [6], [8], [12] and [20]) investigated some interesting and useful properties for analytic functions by applying the Faber polynomial expansion method. Motivated by these and other recent works (see, for example, [1], [14] and [30]), here we make use of the $q$-analysis in order to define new subclasses of analytic and bi-univalent 
functions in $\mathbb{U}$ and (by means of the Faber polynomial expansion method) we determine estimates for the general coefficient $\left|a_{n}\right| \quad(n \geqq 3)$ in the Taylor-Maclaurin series expansion (1.1) of functions in each of these subclasses.

We begin by recalling here some basic definitions and other concept details of the $q$-calculus $(0<q<1)$, which will be used in this paper.

Definition 1.1. Let $q \in(0,1)$ and define the $q$-number $[\kappa]_{q}$ by

$$
[\kappa]_{q}= \begin{cases}\frac{1-q^{\kappa}}{1-q} & (\kappa \in \mathbb{C}) \\ \sum_{k=0}^{n-1} q^{k}=1+q+q^{2}+\cdots+q^{n-1} & (\kappa=n \in \mathbb{N}),\end{cases}
$$

where $\mathbb{N}$ denotes the set of positive integers and $\mathbb{N}_{0}:=\mathbb{N} \cup\{0\}$.

Definition 1.2. Let $q \in(0,1)$ and define the $q$-factorial $[n]_{q}$ ! by

$$
[n]_{q} != \begin{cases}1 & (n=0) \\ \prod_{k=1}^{n}[k]_{q} & (n \in \mathbb{N}) .\end{cases}
$$

Definition 1.3. (see [15] and [16]) The $q$-derivative (or the $q$-difference) $D_{q} f$ of a function $f$ is defined, in a given subset of $\mathbb{C}$, by

$$
\left(D_{q} f\right)(z)= \begin{cases}\frac{f(z)-f(q z)}{(1-q) z} & (z \neq 0) \\ f^{\prime}(0) & (z=0)\end{cases}
$$

provided that $f^{\prime}(0)$ exists.

We note from Definition 1.3 that

$$
\lim _{q \rightarrow 1-}\left(D_{q} f\right)(z)=\lim _{q \rightarrow 1-} \frac{f(z)-f(q z)}{(1-q) z}=f^{\prime}(z)
$$

for a function $f$ which is differentiable in a given subset of $\mathbb{C}$. It is readily deduced from (1.1) and (1.4) that

$$
\left(D_{q} f\right)(z)=1+\sum_{n=2}^{\infty}[n]_{q} a_{n} z^{n-1}
$$

Definition 1.4. The $q$-Pochhammer symbol $[\kappa]_{n, q} \quad\left(\kappa \in \mathbb{C} ; n \in \mathbb{N}_{0}\right)$ is defined as follows:

$$
[\kappa]_{n, q}=\frac{\left(q^{\kappa} ; q\right)_{n}}{(1-q)^{n}}:= \begin{cases}1 & (n=0) \\ {[\kappa]_{q}[\kappa+1]_{q}[\kappa+2]_{q} \cdots[\kappa+n-1]_{q}} & (n \in \mathbb{N}) .\end{cases}
$$

Moreover, the $q$-gamma function $\Gamma_{q}(z)$ is defined by the following recurrence relation:

$$
\Gamma_{q}(z+1)=[z]_{q} \Gamma_{q}(z) \quad \text { and } \quad \Gamma_{q}(1)=1 .
$$


Definition 1.5. [17] For $f \in \mathcal{A}$, let the Ruscheweyh $q$-derivative operator be defined as follows:

where

$$
\mathcal{I}_{q}^{\lambda} f(z)=f(z) * \mathcal{F}_{q, \lambda+1}(z) \quad(z \in \mathbb{U} ; \lambda>-1),
$$

$$
\mathcal{F}_{q, \lambda+1}(z)=z+\sum_{n=2}^{\infty} \frac{\Gamma_{q}(\lambda+n)}{[n-1]_{q} ! \Gamma_{q}(\lambda+1)} z^{n}=z+\sum_{n=2}^{\infty} \frac{[\lambda+1]_{q, n-1}}{[n-1]_{q} !} z^{n}
$$

in terms the Hadamard product (or convolution) given by (1.2).

We next define a certain $q$-integral operator by using the same technique as that used by Noor [19].

Definition 1.6. For $f \in \mathcal{A}$, let the $q$-integral operator $\mathcal{F}_{q, \lambda}$ be defined by

$$
\mathcal{F}_{q, \lambda+1}^{-1}(z) * \mathcal{F}_{q, \lambda+1}(z)=z D_{q} f(z) .
$$

Then

$$
\begin{aligned}
\mathcal{I}_{q}^{\lambda} f(z) & =f(z) * \mathcal{F}_{q, \lambda+1}^{-1}(z) \\
& =z+\sum_{n=2}^{\infty} \Psi_{n-1} a_{n} z^{n} \quad(z \in \mathbb{U} ; \lambda>-1)
\end{aligned}
$$

where

and

$$
\mathcal{F}_{q, \lambda+1}^{-1}(z)=z+\sum_{n=2}^{\infty} \Psi_{n-1} z^{n}
$$

$$
\Psi_{n-1}=\frac{[n]_{q} ! \Gamma_{q}(\lambda+1)}{\Gamma_{q}(\lambda+n)}=\frac{[n]_{q} !}{[\lambda+1]_{q, n-1}} .
$$

Clearly, we have

$$
\mathcal{I}_{q}^{0} f(z)=z D_{q} f(z) \quad \text { and } \quad \mathcal{I}_{q}^{1} f(z)=f(z) .
$$

We note also that, in the limit case when $q \rightarrow 1-$, the $q$-integral operator $\mathcal{F}_{q, \lambda}$ given by Definition 1.6 would reduce to the integral operator which was studied by Noor $[18]$.

The following identity can be easily verified:

$$
z D_{q}\left(\mathcal{I}_{q}^{\lambda+1} f(z)\right)=\left(1+\frac{[\lambda]_{q}}{q^{\lambda}}\right) \mathcal{I}_{q}^{\lambda} f(z)-\frac{[\lambda]_{q}}{q^{\lambda}} \mathcal{I}_{q}^{\lambda+1} f(z) .
$$

When $q \rightarrow 1-$, this last identity (1.6) implies that

$$
z\left(\mathcal{I}^{\lambda+1} f(z)\right)^{\prime}=(1+\lambda) \mathcal{I}^{\lambda} f(z)-\lambda \mathcal{I}^{\lambda+1} f(z),
$$

which is the well-known recurrence relation for the above-mentioned integral operator which studied by Noor [18].

The above-defined $q$-calculus provides valuable tools that have been extensively used in order to examine several subclasses of $\mathcal{A}$. Even though Ismail et al. [14] were the first to use the $q$-derivative operator $D_{q}$ in order to study a certain $q$ analogue of the class $\mathcal{S}^{*}$ of starlike functions in $\mathbb{U}$, yet a rather significant usage of the $q$-calculus in the context of Geometric Function Theory of Complex Analysis was 
basically furnished and the basic (or $q$-) hypergeometric functions were first used in Geometric Function Theory in a book chapter by Srivastava (see, for details, [21, pp. 347 et seq.]; see also [23]).

We now introduce the following subclasses of the analytic and bi-univalent function class $\Sigma$.

Definition 1.7. For a function $f \in \Sigma$, we say that

$$
f \in \mathcal{R}_{q}(\Sigma, \alpha, \gamma) \quad(0 \leqq \alpha<1 ; \gamma \geqq 0)
$$

if and only if

$$
\left|D_{q} f(z)+\gamma z D_{q}^{2} f(z)-\frac{1-\alpha q}{1-q}\right|<\frac{1-\alpha}{1-q} \quad(z \in \mathbb{U})
$$

and

$$
\left|D_{q} g(w)+\gamma w D_{q}^{2} g(w)-\frac{1-\alpha q}{1-q}\right|<\frac{1-\alpha}{1-q} \quad(w \in \mathbb{U}) .
$$

Equivalently, by using the principle of subordination between analytic functions, we can write the above conditions as follows (see, for details, [30]):

$$
D_{q} f(z)+\gamma z D_{q}^{2} f(z) \prec \frac{1+[1-\alpha(1+q)] z}{1-q z} \quad(z \in \mathbb{U})
$$

and

$$
D_{q} g(w)+\gamma w D_{q}^{2} g(w) \prec \frac{1+[1-\alpha(1+q)] w}{1-q w} \quad(w \in \mathbb{U}),
$$

respectively, where $g(w)=f^{-1}(w)$ is given by (1.3).

Definition 1.8. For a function $f \in \Sigma$, we say that

$$
f \in \mathcal{R}_{q}(\Sigma, \alpha, \gamma, \lambda) \quad(0 \leqq \alpha<1 ; \gamma \geqq 0 ; \lambda \geqq 0)
$$

if and only if

$$
D_{q} \mathcal{I}_{q}^{\lambda} f(z)+\gamma z D_{q}^{2} \mathcal{I}_{q}^{\lambda} f(z) \prec \frac{1+[1-\alpha(1+q)] z}{1-q z} \quad(z \in \mathbb{U})
$$

and

$$
D_{q} \mathcal{I}_{q}^{\lambda} g(w)+\lambda w D_{q}^{2} \mathcal{I}_{q}^{\lambda} g(w) \prec \frac{1+[1-\alpha(1+q)] w}{1-q w} \quad(w \in \mathbb{U}),
$$

where $g(w)=f^{-1}(w)$ is given by (1.3).

\section{The Faber polynomial expansion method and its applications}

In this section, by using the Faber polynomial expansion of a function $f \in \mathcal{A}$ of the form (1.1), we observe that the coefficients of its inverse map $g=f^{-1}$ may be expressed as follows (see [4]; see also [13] and [26]):

$$
g(w)=f^{-1}(w)=w+\sum_{n=2}^{\infty} \frac{1}{n} K_{n-1}^{-n}\left(a_{2}, a_{3}, \cdots, a_{n}\right) w^{n},
$$


where

$$
\begin{aligned}
K_{n-1}^{-n}= & \frac{(-n) !}{(-2 n+1) !(n-1) !} a_{2}^{n-1}+\frac{(-n) !}{(2(-n+1)) !(n-3) !} a_{2}^{n-3} a_{3} \\
& +\frac{(-n) !}{(-2 n+3) !(n-4) !} a_{2}^{n-4} a_{4} \\
& +\frac{(-n) !}{(2(-n+2)) !(n-5) !} a_{2}^{n-5}\left[a_{5}+(-n+2) a_{3}^{2}\right] \\
& +\frac{(-n) !}{(-2 n+5) !(n-6) !} a_{2}^{n-6}\left[a_{6}+(-2 n+5) a_{3} a_{4}\right] \\
& +\sum_{j \geqq 7} a_{2}^{n-j} V_{j} .
\end{aligned}
$$

Here, and in what follows, such expressions as (for example) $(-n)$ ! occurring in $(2.2)$ are to be interpreted symbolically by

$$
(-n) ! \equiv \Gamma(1-n):=(-n)(-n-1)(-n-2) \cdots \quad\left(n \in \mathbb{N}_{0}\right)
$$

and $V_{j}(7 \leqq j \leqq n)$ is a homogeneous polynomial in the variables $a_{2}, a_{3}, \cdots, a_{n}$.

In particular, the first three terms of $K_{n-1}^{-n}$ are given below:

$$
K_{1}^{-2}=-2 a_{2}, \quad K_{2}^{-3}=3\left(2 a_{2}^{2}-a_{3}\right)
$$

and

$$
K_{3}^{-4}=-4\left(5 a_{2}^{3}-5 a_{2} a_{3}+a_{4}\right) .
$$

In general, an expansion of $K_{n}^{\mathfrak{p}}$ is given by (see, for details, [3])

$$
K_{n}^{\mathfrak{p}}=\mathfrak{p} a_{n}+\frac{\mathfrak{p}(\mathfrak{p}-1)}{2} E_{n}^{2}+\frac{\mathfrak{p} !}{(-3) ! 3 !} E_{n}^{3}+\cdots+\frac{\mathfrak{p} !}{(\mathfrak{p}-n) ! n !} E_{n}^{n} \quad(\mathfrak{p} \in \mathbb{Z}),
$$

where $\mathbb{Z}:=\{0, \pm 1, \pm 2, \cdots\}$ and

$$
E_{n}^{\mathfrak{p}}=E_{n}^{\mathfrak{p}}\left(a_{2}, a_{3}, \cdots\right) .
$$

It is clearly seen that

$$
E_{n}^{n}\left(a_{1}, a_{2}, \cdots, a_{n}\right)=a_{1}^{n}
$$

and

$$
E_{n-1}^{m}\left(a_{2}, \cdots, a_{n}\right)=\sum_{n=2}^{\infty} \frac{m !\left(a_{2}\right)^{\mu_{1}} \cdots\left(a_{n}\right)^{\mu_{n-1}}}{\mu_{1 !}, \cdots, \mu_{n-1} !} \quad(m \leqq n) .
$$

We also have (see [2])

$$
E_{n-1}^{n-1}\left(a_{2}, \cdots, a_{n}\right)=a_{2}^{n-1}
$$

and

$$
E_{n}^{m}\left(a_{1}, a_{2}, \cdots, a_{n}\right)=\sum\left(\frac{m !}{\mu_{1} ! \cdots \mu_{n} !}\right) a_{1}^{\mu_{1}} \cdots a_{n}^{\mu_{n}} \quad(m \leqq n),
$$

where $a_{1}=1$ and the sum is taken over all non-negative integers $\mu_{1}, \cdots, \mu_{n}$ satisfying the following conditions:

$$
\mu_{1}+\mu_{2}+\cdots+\mu_{n}=m
$$

and

$$
\mu_{1}+2 \mu_{2}+\cdots+n \mu_{n}=n
$$


By a similar argument, we note that

$$
E_{n}^{n}\left(a_{1}, \cdots, a_{n}\right)=E_{1}^{n}
$$

and that the first and the last polynomials are given by

$$
E_{n}^{n}=a_{1}^{n} \quad \text { and } \quad E_{n}^{1}=a_{n} .
$$

We now state and prove our main results. Throughout our discussion, the parameters $\mathcal{L}$ and $\mathcal{M}$ are given by

$$
\mathcal{L}:=[1-\alpha(1+q)] \quad \text { and } \quad \mathcal{M}:=-q .
$$

Theorem 2.1. For $0 \leqq \alpha<1$ and $\gamma \geqq 0$, let $f \in \mathcal{R}_{q}(\Sigma, \alpha, \gamma)$. If

$$
a_{m}=0 \quad(2 \leqq m \leqq n-1),
$$

then

$$
\left|a_{n}\right| \leqq \frac{|1-\alpha+q(1-\alpha)|}{[n]_{q}+\gamma[n]_{q}[n-1]_{q}} \quad(n \geqq 3) .
$$

Proof. For the function $f \in \mathcal{R}_{q}(\Sigma, \alpha, \gamma)$ of the form (1.1), we have

$$
D_{q} f(z)+\gamma z D_{q}^{2} f(z)=1+\sum_{n=2}^{\infty}\left([n]_{q}+\gamma[n]_{q}[n-1]_{q}\right) a_{n} z^{n-1}
$$

and, for its inverse map $g=f^{-1}$, we get

$$
D_{q} g(w)+\gamma w D_{q}^{2} g(w)=1+\sum_{n=2}^{\infty}\left([n]_{q}+\gamma[n]_{q}[n-1]_{q}\right) b_{n} w^{n-1},
$$

where

$$
b_{n}=\frac{1}{[n]_{q}} K_{n-1}^{-n}\left(a_{2}, a_{3}, \cdots, a_{n}\right) .
$$

Since both the function $f$ and its inverse map $g=f^{-1}$ are in $\mathcal{R}_{q}(\Sigma, \alpha, \gamma)$, by the definition of subordination, there exist two Schwarz functions $p(z)$ and $q(w)$ given by

$$
p(z)=\sum_{n=1}^{\infty} c_{n} z^{n} \quad \text { and } \quad q(w)=\sum_{n=1}^{\infty} d_{n} w^{n} \quad(z, w \in \mathbb{U}),
$$

so that we have

$$
\begin{aligned}
D_{q} f(z)+\gamma z D_{q}^{2} f(z) & =\frac{1+\mathcal{L} p(z)}{1+\mathcal{M} p(z)} \\
& =1-\sum_{n=1}^{\infty}(\mathcal{L}-\mathcal{M}) K_{n}^{-1}\left(c_{1}, c_{2}, \cdots, c_{n}, \mathcal{M}\right) z^{n}
\end{aligned}
$$

and

$$
\begin{aligned}
D_{q} g(w)+\gamma w D_{q}^{2} g(w) & =\frac{1+\mathcal{L} q(w)}{1+\mathcal{M} q(w)} \\
& =1-\sum_{n=1}^{\infty}(\mathcal{L}-\mathcal{M}) K_{n}^{-1}\left(d_{1}, d_{2}, \cdots, d_{n}, \mathcal{M}\right) w^{n}
\end{aligned}
$$


In general, for any $\mathfrak{p} \in \mathbb{N}$ and $n \geqq 2$, we have the following expansion of $K_{n}^{\mathfrak{p}}\left(k_{1}, k_{2}, \cdots, k_{n}, \mathcal{M}\right)$ (see [3] and [4]):

$$
\begin{aligned}
K_{n}^{\mathfrak{p}}( & \left.k_{1}, k_{2}, \cdots, k_{n}, \mathcal{M}\right) \\
= & \frac{\mathfrak{p} !}{(\mathfrak{p}-n) ! n !} k_{1}^{n} \mathcal{M}^{n-1}+\frac{\mathfrak{p} !}{(\mathfrak{p}-n+1) !(n-2) !} k_{1}^{n-2} k_{2} \mathcal{M}^{n-2} \\
& +\frac{\mathfrak{p} !}{(\mathfrak{p}-n+2) !(n-3) !} \cdot k_{1}^{n-3} k_{3} \mathcal{M}^{n-3} \\
& +\frac{\mathfrak{p} !}{(\mathfrak{p}-n+3) !(n-4) !} k_{1}^{n-4}\left[k_{4} \mathcal{M}^{n-4}+\frac{\mathfrak{p}-n+3}{2} k_{3}^{2} \mathcal{M}\right] \\
& +\frac{\mathfrak{p} !}{(\mathfrak{p}-n+4) !(n-5) !} k_{1}^{n-5}\left[k_{5} \mathcal{M}^{n-5}+(\mathfrak{p}-n+4) k_{3} k_{4} \mathcal{M}\right] \\
& +\sum_{j \geqq 6} k_{1}^{n-1} X_{j},
\end{aligned}
$$

where $X_{j}$ is a homogeneous polynomial of degree $j$ in the variables $k_{1}, k_{2}, \cdots, k_{n}$. For the coefficients of the Schwarz functions $p(z)$ and $q(w)$, we have (see [10])

$$
\left|c_{n}\right| \leqq 1 \quad \text { and } \quad\left|d_{n}\right| \leqq 1
$$

Thus, upon comparing with the corresponding coefficients in (2.4) and (2.6), we find that

$$
\left([n]_{q}+\gamma[n]_{q}[n-1]_{q}\right) a_{n}=-(\mathcal{L}-\mathcal{M}) K_{n-1}^{-1}\left(c_{1}, c_{2}, \cdots, c_{n-1}, \mathcal{M}\right) .
$$

Similarly, in view of the corresponding coefficients in (2.5) and (2.7), we have

$$
\left([n]_{q}+\gamma[n]_{q}[n-1]_{q}\right) b_{n}=-(\mathcal{L}-\mathcal{M}) K_{n}^{-1}\left(d_{1}, d_{2}, \cdots, d_{n}, \mathcal{M}\right) .
$$

We note for

$$
a_{m}=0 \quad(2 \leqq m \leqq n-1) \quad \text { and } \quad b_{n}=-a_{n},
$$

that

$$
\left([n]_{q}+\gamma[n]_{q}[n-1]_{q}\right) a_{n}=-(\mathcal{L}-\mathcal{M}) c_{n-1}
$$

and

$$
-\left([n]_{q}+\gamma[n]_{q}[n-1]_{q}\right) a_{n}=-(\mathcal{L}-\mathcal{M}) d_{n-1} .
$$

Taking the moduli in (2.11) and (2.12), we thus obtain

$$
\begin{aligned}
\left|a_{n}\right| & \leqq \frac{|\mathcal{L}-\mathcal{M}|}{[n]_{q}+\gamma[n]_{q}[n-1]_{q}}\left|c_{n-1}\right| \\
& =\frac{|\mathcal{L}-\mathcal{M}|}{[n]_{q}+\gamma[n]_{q}[n-1]_{q}}\left|d_{n-1}\right| .
\end{aligned}
$$

Therefore, we have

$$
\left|a_{n}\right| \leqq \frac{|1-\alpha+q(1-\alpha)|}{[n]_{q}+\gamma[n]_{q}[n-1]_{q}} \quad(n \geqq 3),
$$

which completes the proof of the assertion (2.3) of Theorem 2.1. 
If we let $q \rightarrow 1-$ in Theorem 2.1 above, we obtain the following known result given by Srivastava et al. [26].

Corollary 2.2. (see [26]) Let $f$ given by (1.1) be in the class

$$
\mathcal{R}_{\Sigma}^{\alpha, \gamma}(0 \leqq \alpha<1 ; \gamma \geqq 0) .
$$

If

$$
a_{m}=0 \quad(2 \leqq m \leqq n-1),
$$

then

$$
\left|a_{n}\right| \leqq \frac{2(1-\alpha)}{n[1+\gamma(n-1)]} \quad(n \in \mathbb{N} \backslash\{1,2\}) .
$$

Theorem 2.3. For $0 \leqq \alpha<1$ and $0 \leqq \gamma$, let $f \in \mathcal{R}_{q}(\Sigma, \alpha, \gamma)$. Then

$$
\begin{gathered}
\left|a_{2}\right| \leqq \min \left\{\frac{|1-\alpha+q(1-\alpha)|}{[2]_{q}+\gamma[2]_{q}[1]_{q}}, \sqrt{\left.\frac{2(1+q)|1-\alpha+q(1-\alpha)|}{[2]_{q}\left([3]_{q}+\gamma[3]_{q}[2]_{q}\right)}\right\},}\right. \\
\left|a_{3}\right| \leqq \min \left\{\frac{|1-\alpha+q(1-\alpha)|}{[1]_{q}+[1]_{q}}\left(\frac{[2]_{q}|1-\alpha+q(1-\alpha)|}{\left([2]_{q}+\gamma[2]_{q}[1]_{q}\right)^{2}}+\frac{2}{[3]_{q}+\gamma[3]_{q}[2]_{q}}\right),\right. \\
\left.\frac{2(q+2)|1-\alpha+q(1-\alpha)|}{\left([1]_{q}+[1]_{q}\right)\left([3]_{q}+\gamma[3]_{q}[2]_{q}\right)}\right\}, \\
\left|a_{3}-[2]_{q} a_{2}^{2}\right| \leqq \frac{(1+q)|1-\alpha+q(1-\alpha)|}{[3]_{q}+\gamma[3]_{q}[2]_{q}}
\end{gathered}
$$

and

$$
\left|a_{3}-\frac{[2]_{q}}{[1]_{q}+[1]_{q}} a_{2}^{2}\right| \leqq \frac{2|1-\alpha+q(1-\alpha)|}{\left|\left([1]_{q}+[1]_{q}\right)\left([3]_{q}+\gamma[3]_{q}[2]_{q}\right)\right|}
$$

Proof. Upon setting $n=2$ and $n=3$ in (2.9) and (2.10), respectively, we get

$$
\begin{gathered}
\left([2]_{q}+\gamma[2]_{q}[1]_{q}\right) a_{2}=-(\mathcal{L}-\mathcal{M}) c_{1} \\
\left([3]_{q}+\gamma[3]_{q}[2]_{q}\right) a_{3}=-(\mathcal{L}-\mathcal{M})\left(\mathcal{M} c_{1}^{2}-c_{2}\right) \\
-\left([2]_{q}+\gamma[2]_{q}[1]_{q}\right) a_{2}=-(\mathcal{L}-\mathcal{M}) d_{1}
\end{gathered}
$$

and

$$
\left([3]_{q}+\gamma[3]_{q}[2]_{q}\right)\left([2]_{q} a_{2}^{2}-a_{3}\right)=-(\mathcal{L}-\mathcal{M})\left(\mathcal{M} d_{1}^{2}-d_{2}\right)
$$


From (2.13) and (2.15), we have

$$
\begin{aligned}
\left|a_{2}\right| & \leqq \frac{|\mathcal{L}-\mathcal{M}|}{[2]_{q}+\gamma[2]_{q}[1]_{q}}\left|c_{1}\right| \\
& =\frac{|\mathcal{L}-\mathcal{M}|}{[2]_{q}+\gamma[2]_{q}[1]_{q}}\left|d_{1}\right| \\
& \leqq \frac{|1-\alpha+q(1-\alpha)|}{[2]_{q}+\gamma[2]_{q}[1]_{q}} .
\end{aligned}
$$

Adding (2.14) and (2.16), we find that

$$
[2]_{q}\left([3]_{q}+\gamma[3]_{q}[2]_{q}\right) a_{2}^{2}=-(\mathcal{L}-\mathcal{M})\left[\mathcal{M}\left(c_{1}^{2}+d_{1}^{2}\right)-\left(c_{2}+d_{2}\right)\right],
$$

which, upon taking the moduli on both sides, yields

$$
\left|a_{2}\right|^{2}=\frac{2|\mathcal{L}-\mathcal{M}|(|\mathcal{M}|+1)}{[2]_{q}\left([3]_{q}+\gamma[3]_{q}[2]_{q}\right)} .
$$

This last equation can be written as follows:

$$
\left|a_{2}\right| \leqq \sqrt{\frac{2(1+q)|1-\alpha+q(1-\alpha)|}{[2]_{q}\left([3]_{q}+\gamma[3]_{q}[2]_{q}\right)}} .
$$

Now, in order to find $\left|a_{3}\right|$, by subtracting (2.16) from (2.14), we obtain

$$
a_{3}=\frac{(\mathcal{L}-\mathcal{M})\left[\mathcal{M}\left(d_{1}^{2}-c_{1}^{2}\right)-\left(c_{2}-d_{2}\right)\right]}{\left([1]_{q}+[1]_{q}\right)\left([3]_{q}+\gamma[3]_{q}[2]_{q}\right)}+\frac{[2]_{q}}{\left([1]_{q}+[1]_{q}\right)} a_{2}^{2} .
$$

Taking the moduli in (2.20) and using the fact that $d_{1}^{2}=c_{1}^{2}$, we have

$$
\left|a_{3}\right| \leqq \frac{2|\mathcal{L}-\mathcal{M}|}{\left([1]_{q}+[1]_{q}\right)\left([3]_{q}+\gamma[3]_{q}[2]_{q}\right)}+\frac{[2]_{q}}{[1]_{q}+[1]_{q}}\left|a_{2}\right|^{2} .
$$

Using (2.17) in (2.21), we obtain

$$
\begin{aligned}
\left|a_{3}\right| \leqq \frac{|1-\alpha+q(1-\alpha)|}{[1]_{q}+[1]_{q}} & \\
& \cdot\left(\frac{[2]_{q}|1-\alpha+q(1-\alpha)|}{\left([2]_{q}+\gamma[2]_{q}[1]_{q}\right)^{2}}+\frac{2}{[3]_{q}+\gamma[3]_{q}[2]_{q}}\right) .
\end{aligned}
$$

Again, by using the equation (2.19) in (2.21), we have

$$
\left|a_{3}\right| \leqq \frac{2(q+2)|1-\alpha+q(1-\alpha)|}{\left([1]_{q}+[1]_{q}\right)\left([3]_{q}+\gamma[3]_{q}[2]_{q}\right)}
$$

We also find from (2.16) that

$$
\left|a_{3}-[2]_{q} a_{2}^{2}\right| \leqq \frac{(1+q)|1-\alpha+q(1-\alpha)|}{[3]_{q}+\gamma[3]_{q}[2]_{q}} .
$$


From (2.20) and using the fact that $d_{1}^{2}=c_{1}^{2}$, we have

$$
a_{3}-\frac{[2]_{q}}{[1]_{q}+[1]_{q}} a_{2}^{2}=\frac{(\mathcal{L}-\mathcal{M})\left(c_{2}-d_{2}\right)}{\left([1]_{q}+[1]_{q}\right)\left([3]_{q}+\gamma[3]_{q}[2]_{q}\right)} .
$$

Finally, by taking the moduli in (2.24), we finally obtain

$$
\left|a_{3}-\frac{[2]_{q}}{[1]_{q}+[1]_{q}} a_{2}^{2}\right| \leqq \frac{2|1-\alpha+q(1-\alpha)|}{\left|\left([1]_{q}+[1]_{q}\right)\left([3]_{q}+\gamma[3]_{q}[2]_{q}\right)\right|}
$$

The proof of Theorem 2.3 is thus completed.

In the limit case when $q \rightarrow 1-$, Theorem 2.3 yields the following bounds on $\left|a_{2}\right|$ and $\left|a_{3}\right|$ given by Srivastava et al. [26].

Corollary 2.4. (see [26]) Let $f$ given by (1.1) be in the class

$$
\mathcal{R}_{\Sigma}^{\alpha, \gamma}(0 \leqq \alpha<1 ; \gamma \geqq 0)
$$

Then

$$
a_{2} \leqq \begin{cases}\sqrt{\frac{2(1-\alpha)}{3(1+2 \gamma)}} & \left(0 \leqq \alpha \leqq \frac{1+2 \gamma-2 \gamma^{2}}{3(1+2 \gamma)}\right) \\ \frac{1-\alpha}{1+\gamma} & \left(\frac{1+2 \gamma-2 \gamma^{2}}{3(1+2 \gamma)} \leqq \alpha<1\right)\end{cases}
$$

and

$$
a_{3} \leqq \frac{2(1-\alpha)}{3(1+2 \gamma)}
$$

Theorem 2.5. For $0 \leqq \alpha<1$ and $0 \leqq \gamma$, let $f \in \mathcal{R}_{q}(\Sigma, \alpha, \gamma, \lambda)$. If

$$
a_{m}=0 \quad(2 \leqq m \leqq n-1),
$$

then

$$
\left|a_{n}\right| \leqq \frac{|1-\alpha+q(1-\alpha)|[\lambda+1]_{q, n-1}}{\left([n]_{q}+\gamma[n]_{q}[n-1]_{q}\right)[n]_{q} !} \quad(n \geqq 3) .
$$

Proof. For the function $f \in \mathcal{R}_{q}(\Sigma, \alpha, \gamma, \lambda)$ of the form (1.1), we have

$$
\begin{aligned}
D_{q} \mathcal{I}_{q}^{\lambda} f(z) & +\gamma z D_{q}^{2} \mathcal{I}_{q}^{\lambda} f(z) \\
= & 1+\sum_{n=2}^{\infty}\left([n]_{q}+\gamma[n]_{q}[n-1]_{q}\right) \Psi_{n-1} a_{n} z^{n-1} .
\end{aligned}
$$

Also, for its inverse mapping $g=f^{-1}$, we have

$$
\begin{aligned}
D_{q} \mathcal{I}_{q}^{\lambda} g(w) & +\gamma w D_{q}^{2} \mathcal{I}_{q}^{\lambda} g(w) \\
= & 1+\sum_{n=2}^{\infty}\left([n]_{q}+\gamma[n]_{q}[n-1]_{q}\right) \Psi_{n-1} b_{n} w^{n-1}
\end{aligned}
$$


where

$$
b_{n}=\frac{1}{[n]_{q}} K_{n-1}^{-n}\left(a_{2}, a_{3}, \cdots, a_{n}\right) .
$$

Since, both $f$ and its inverse $g=f^{-!}$are in the function class $\mathcal{R}_{q}(\Sigma, \alpha, \gamma, \lambda)$, by the definition of subordination, there exist two Schwarz functions $p(z)$ and $q(w)$ given by

$$
p(z)=\sum_{n=1}^{\infty} c_{n} z^{n} \quad \text { and } \quad q(w)=\sum_{n=1}^{\infty} d_{n} w^{n} \quad(z, w \in \mathbb{U}),
$$

so that we have

$$
\begin{aligned}
D_{q} \mathcal{I}_{q}^{\lambda} f(z) & +\gamma z D_{q}^{2} \mathcal{I}_{q}^{\lambda} f(z) \\
& =\frac{1+\mathcal{L} p(z)}{1+\mathcal{M} p(z)} \\
& =1-\sum_{n=1}^{\infty}(\mathcal{L}-\mathcal{M}) K_{n}^{-1}\left(c_{1}, c_{2}, \cdots, c_{n}, \mathcal{M}\right) z^{n}
\end{aligned}
$$

and

$$
\begin{aligned}
D_{q} \mathcal{I}_{q}^{\lambda} g(w) & +\gamma w D_{q}^{2} \mathcal{I}_{q}^{\lambda} g(w) \\
& =\frac{1+\mathcal{L} q(w)}{1+\mathcal{M} q(w)} \\
& =1-\sum_{n=1}^{\infty}(\mathcal{L}-\mathcal{M}) K_{n}^{-1}\left(d_{1}, d_{2}, \cdots, d_{n}, \mathcal{M}\right) w^{n}
\end{aligned}
$$

In general, for any $\mathfrak{p} \in \mathbb{N}$ and $n \geqq 2$, an expansion of

$$
K_{n}^{\mathfrak{p}}\left(k_{1}, k_{2}, \cdots, k_{n}, \mathcal{M}\right)
$$

is given by (2.8) (see [3] and [4]). Moreover, the coefficients of the Schwarz functions $p(z)$ and $q(w)$ are constrained by (see $[10]$ )

$$
\left|c_{n}\right| \leqq 1 \quad \text { and } \quad\left|d_{n}\right| \leqq 1
$$

Thus, upon comparing the corresponding coefficients in (2.26) and (2.28), we find that

$$
\begin{aligned}
\left([n]_{q}+\gamma\right. & {\left.[n]_{q}[n-1]_{q}\right) \Psi_{n-1} a_{n} } \\
& =-(\mathcal{L}-\mathcal{M}) K_{n-1}^{-1}\left(c_{1}, c_{2}, \cdots, c_{n-1}, \mathcal{M}\right) .
\end{aligned}
$$

Similarly, by comparing the corresponding coefficients in (2.27) and (2.29), we have

$$
\begin{aligned}
\left([n]_{q}+\gamma\right. & {\left.[n]_{q}[n-1]_{q}\right) \Psi_{n-1} b_{n} } \\
& =-(\mathcal{L}-\mathcal{M}) K_{n}^{-1}\left(d_{1}, d_{2}, \cdots, d_{n}, \mathcal{M}\right) .
\end{aligned}
$$

We note also that, for

$$
a_{m}=0 \quad(2 \leqq m \leqq n-1) \quad \text { and } \quad b_{n}=-a_{n},
$$

we have

$$
\left([n]_{q}+\gamma[n]_{q}[n-1]_{q}\right) \Psi_{n-1} a_{n}=-(\mathcal{L}-\mathcal{M}) c_{n-1}
$$


and

$$
-\left([n]_{q}+\gamma[n]_{q}[n-1]_{q}\right) \Psi_{n-1} a_{n}=-(\mathcal{L}-\mathcal{M}) d_{n-1} .
$$

Finally, by taking the moduli in (2.32) and (2.33), we obtain

$$
\begin{aligned}
\left|a_{n}\right| & \leqq \frac{|\mathcal{L}-\mathcal{M}|}{\left([n]_{q}+\gamma[n]_{q}[n-1]_{q}\right) \Psi_{n-1}}\left|c_{n-1}\right| \\
& =\frac{|\mathcal{L}-\mathcal{M}|}{\left([n]_{q}+\gamma[n]_{q}[n-1]_{q}\right) \Psi_{n-1}}\left|d_{n-1}\right|
\end{aligned}
$$

Consequently, we have

$$
\left|a_{n}\right| \leqq \frac{|1-\alpha+q(1-\alpha)|[\lambda+1]_{q, n-1}}{\left([n]_{q}+\gamma[n]_{q}[n-1]_{q}\right)[n]_{q} !} \quad(n \geqq 3),
$$

which completes the proof of the assertion (2.25) of Theorem 2.5.

Theorem 2.6. For $0 \leqq \alpha<1$ and $\gamma \geqq 0$, let $f \in \mathcal{R}_{q}(\Sigma, \alpha, \gamma, \lambda)$. Then

$$
\begin{aligned}
& \left|a_{2}\right| \leqq \min \left\{\frac{|1-\alpha+q(1-\alpha)|[\lambda+1]_{q, 1}}{\left([2]_{q}+\gamma[2]_{q}[1]_{q}\right)[2]_{q} !}\right. \\
& \left.\sqrt{\frac{2(1+q)|1-\alpha+q(1-\alpha)|[\lambda+1]_{q, 2}}{[2]_{q}\left([3]_{q}+\gamma[3]_{q}[2]_{q}\right)[3]_{q} !}}\right\} \\
& \left|a_{3}\right| \leqq \min \left\{\frac { | 1 - \alpha + q ( 1 - \alpha ) | } { [ 1 ] _ { q } + [ 1 ] _ { q } } \left(\frac{\left([\lambda+1]_{q, 1}\right)^{2}[2]_{q}|1-\alpha+q(1-\alpha)|}{\left([2]_{q} !\right)^{2}\left([2]_{q}+\gamma[2]_{q}[1]_{q}\right)^{2}}\right.\right. \\
& \left.+\frac{2[\lambda+1]_{q, 2}}{\left([3]_{q}+\gamma[3]_{q}[2]_{q}\right)[3]_{q} !}\right) \\
& \left.\frac{2(q+2)|1-\alpha+q(1-\alpha)|[\lambda+1]_{q, 2}}{\left([1]_{q}+[1]_{q}\right)\left([3]_{q}+\gamma[3]_{q}[2]_{q}\right)[3]_{q} !}\right\} \text {, } \\
& \left|a_{3}-[2]_{q} a_{2}^{2}\right| \leqq \frac{(1+q)|1-\alpha+q(1-\alpha)|[\lambda+1]_{q, 2}}{\left([3]_{q}+\gamma[3]_{q}[2]_{q}\right)[3]_{q} !}
\end{aligned}
$$

and

$$
\left|a_{3}-\left(\frac{[2]_{q}}{[1]_{q}+[1]_{q}}\right) a_{2}^{2}\right| \leqq \frac{2|1-\alpha+q(1-\alpha)|[\lambda+1]_{q, 2}}{\left|\left([1]_{q}+[1]_{q}\right)\left([3]_{q}+\gamma[3]_{q}[2]_{q}\right)\right|[3]_{q} !}
$$


Proof. Upon setting $n=2$ and $n=3$ in (2.30) and (2.31), respectively, we have

$$
\begin{gathered}
\left([2]_{q}+\gamma[2]_{q}[1]_{q}\right) \Psi_{1} a_{2}=-(\mathcal{L}-\mathcal{M}) c_{1} \\
\left([3]_{q}+\gamma[3]_{q}[2]_{q}\right) \Psi_{2} a_{3}=-(\mathcal{L}-\mathcal{M})\left(\mathcal{M} c_{1}^{2}-c_{2}\right) \\
-\left([2]_{q}+\gamma[2]_{q}[1]_{q}\right) \Psi_{1} a_{2}=-(\mathcal{L}-\mathcal{M}) d_{1}
\end{gathered}
$$

and

$$
\left([3]_{q}+\gamma[3]_{q}[2]_{q}\right) \Psi_{2}\left([2]_{q} a_{2}^{2}-a_{3}\right)=-(\mathcal{L}-\mathcal{M})\left(\mathcal{M} d_{1}^{2}-d_{2}\right) .
$$

Making use of (2.38) and (2.40), we find that

$$
\begin{aligned}
\left|a_{2}\right| & \leqq \frac{|\mathcal{L}-\mathcal{M}|}{\left([2]_{q}+\gamma[2]_{q}[1]_{q}\right) \Psi_{1}}\left|c_{1}\right| \\
& =\frac{|\mathcal{L}-\mathcal{M}|}{\left([2]_{q}+\gamma[2]_{q}[1]_{q}\right) \Psi_{1}}\left|d_{1}\right| \\
& \leqq \frac{|1-\alpha+q(1-\alpha)|[\lambda+1]_{q, 1}}{\left([2]_{q}+\gamma[2]_{q}[1]_{q}\right)[2]_{q} !}
\end{aligned}
$$

Also, by adding (2.39) and (2.41), we have

$$
[2]_{q}\left([3]_{q}+\gamma[3]_{q}[2]_{q}\right) \Psi_{2} a_{2}^{2}=-(\mathcal{L}-\mathcal{M})\left[\mathcal{M}\left(c_{1}^{2}+d_{1}^{2}\right)-\left(c_{2}+d_{2}\right)\right] .
$$

Now, if we take the moduli in both sides of (2.43), we obtain

$$
\left|a_{2}\right|^{2}=\frac{2|\mathcal{L}-\mathcal{M}|(|\mathcal{M}|+1)}{[2]_{q}\left([3]_{q}+\gamma[3]_{q}[2]_{q}\right) \Psi_{2}}
$$

so that

$$
\left|a_{2}\right| \leqq \sqrt{\frac{2(1+q)|1-\alpha+q(1-\alpha)|[\lambda+1]_{q, 2}}{[2]_{q}\left([3]_{q}+\gamma[3]_{q}[2]_{q}\right)[3]_{q} !}}
$$

In order to find $\left|a_{3}\right|$, we subtract (2.41) from (2.39), We thus obtain

$$
a_{3}=\frac{(\mathcal{L}-\mathcal{M})\left[\mathcal{M}\left(d_{1}^{2}-c_{1}^{2}\right)-\left(c_{2}-d_{2}\right)\right]}{\left([1]_{q}+[1]_{q}\right)\left([3]_{q}+\gamma[3]_{q}[2]_{q}\right) \Psi_{2}}+\left(\frac{[2]_{q}}{\left([1]_{q}+[1]_{q}\right)}\right) a_{2}^{2},
$$

which, after taking the moduli and using the fact that

$$
d_{1}^{2}=c_{1}^{2}
$$

yields

$$
\left|a_{3}\right| \leqq \frac{2|\mathcal{L}-\mathcal{M}|}{\left([1]_{q}+[1]_{q}\right)\left([3]_{q}+\gamma[3]_{q}[2]_{q}\right) \Psi_{2}}+\left(\frac{[2]_{q}}{[1]_{q}+[1]_{q}}\right)\left|a_{2}\right|^{2}
$$


Using (2.42) in (2.46), we have

$$
\begin{array}{r}
\left|a_{3}\right| \leqq \frac{|1-\alpha+q(1-\alpha)|}{[1]_{q}+[1]_{q}}\left(\frac{\left([\lambda+1]_{q, 1}\right)^{2}[2]_{q}|1-\alpha+q(1-\alpha)|}{\left([2]_{q} !\right)^{2}\left([2]_{q}+\gamma[2]_{q}[1]_{q}\right)^{2}}\right. \\
\left.+\frac{2[\lambda+1]_{q, 2}}{\left([3]_{q}+\gamma[3]_{q}[2]_{q}\right)[3]_{q} !}\right) .
\end{array}
$$

Again, by using (2.44) in (2.46), we get

$$
\left|a_{3}\right| \leqq \frac{2(q+2)|1-\alpha+q(1-\alpha)|[\lambda+1]_{q, 2}}{\left([1]_{q}+[1]_{q}\right)\left([3]_{q}+\gamma[3]_{q}[2]_{q}\right)[3]_{q} !}
$$

It follows from (2.41) that

$$
\left|a_{3}-[2]_{q} a_{2}^{2}\right| \leqq \frac{(1+q)|1-\alpha+q(1-\alpha)|[\lambda+1]_{q, 2}}{\left([3]_{q}+\gamma[3]_{q}[2]_{q}\right)[3]_{q} !}
$$

Using the fact that

$$
d_{1}^{2}=c_{1}^{2}
$$

in $(2.45)$, we have

$$
a_{3}-\left(\frac{[2]_{q}}{[1]_{q}+[1]_{q}}\right) a_{2}^{2}=\frac{(\mathcal{L}-\mathcal{M})\left(c_{2}-d_{2}\right)}{\left([1]_{q}+[1]_{q}\right)\left([3]_{q}+\gamma[3]_{q}[2]_{q}\right) \Psi_{2}} .
$$

By taking the moduli on both sides of (2.48), we finally obtain

$$
\left|a_{3}-\left(\frac{[2]_{q}}{\left([1]_{q}+[1]_{q}\right)}\right) a_{2}^{2}\right| \leqq \frac{2|1-\alpha+q(1-\alpha)|[\lambda+1]_{q, 2}}{\left|\left([1]_{q}+[1]_{q}\right)\left([3]_{q}+\gamma[3]_{q}[2]_{q}\right)\right|[3]_{q} !}
$$

which completes the proof of Theorem 2.6.

\section{Concluding remarks and observations}

Here, in our present investigation, we have successfully applied the Faber polynomial expansion method as well as the $q$-analysis in our study of several new subclasses of analytic and bi-univalent functions by using a certain $q$-integral operator in the open unit disk $\mathbb{U}$. We have derived bounds for the $n$th coefficient in the Taylor-Maclaurin series expansion for functions in each of these newly-defined analytic and bi-univalent function classes subject to a gap series condition. By means of corollaries of our main theorems, we have also highlighted some known consequences of our main results, which were given recently by Srivastava et al. [26]. 


\section{References}

[1] Agrawal, S., Sahoo, S.K., A generalization of starlike functions of order $\alpha$, Hokkaido Math. J., 46(2017), 15-27.

[2] Airault, H., Symmetric sums associated to the factorization of Grunsky coefficients, in "Proceedings of the Conference on Groups and Symmetries", Montréal, Canada, 2007.

[3] Airault, H., Remarks on Faber polynomials, Internat. Math. Forum, 3(2008), 449-456.

[4] Airault, H., Bouali, A., Differential calculus on the Faber polynomials, Bull. Sci. Math., 130(2006), 179-222.

[5] Altınkaya, Ş., Yalçın, S., Faber polynomial coefficient bounds for a subclass of bi-univalent functions, C.R. Acad. Sci. Paris Sér. I, 353(2015), 1075-1080.

[6] Altınkaya, Ş., Yalçın, S., Faber polynomial coefficient bounds for a subclass of bi-univalent functions, Stud. Univ. Babeş-Bolyai Math., 61(2016), 37-44.

[7] Bulut, S., Faber polynomial coefficient estimates for a comprehensive subclass of analytic bi-univalent functions, C. R. Acad. Sci. Paris Sér. I, 352(2014), 479-484.

[8] Bulut, S., Faber polynomial coefficient estimates for certain subclasses of meromorphic bi-univalent functions, C.R. Acad. Sci. Paris Sér. I, 353(2015), 113-116.

[9] Çağlar, M., Deniz, E., Srivastava, H.M., Second Hankel determinant for certain subclasses of bi-univalent functions, Turkish J. Math., 41(2017), 694-706.

[10] Duren, P.L., Univalent Functions, Grundlehren der Mathematischen Wissenschaften, Band 259, Springer-Verlag, New York, Berlin, Heidelberg and Tokyo, 1983.

[11] Faber, G., Über polynomische Entwicklungen, Math. Ann., 57(1903), 389-408.

[12] Hamidi, S.G., Jahangiri, J.M., Faber polynomial coefficient estimates for analytic biclose-to-convex functions, C.R. Acad. Sci. Paris Sér. I, 352(2014), 17-20.

[13] Hamidi, S.G., Jahangiri, J.M., Faber polynomial coefficients of bi-subordinate functions, C.R. Acad. Sci. Paris Sér. I, 354(2016), 365-370.

[14] Ismail, M.E.H., Merkes, E., Styer, D., A generalization of starlike functions, Complex Variables Theory Appl., 14(1990), 77-84.

[15] Jackson, F.H., On q-definite integrals, Quart. J. Pure Appl. Math., 41(1910), 193-203.

[16] Jackson, F.H., q-difference equations, Amer. J. Math., 32(1910), 305-314.

[17] Kanas, S., Răducanu, D., Some class of analytic functions related to conic domains, Math. Slovaca, 64(2014), 1183-1196.

[18] Noor, K. I., On new classes of integral operators, J. Natur. Geom., 16(1999), 71-80.

[19] Noor, K.I., Noor, M.A., On certain classes of analytic functions defined by Noor integral operator, J. Math. Anal. Appl., 281(2003), 244-252.

[20] Sharma, P., Faber polynomial coefficient estimates for a class of analytic bi-univalent functions involving a certain differential operator, Asian-European J. Math., 10(2017), Article ID 1750016, 1-11.

[21] Srivastava, H.M., Univalent functions, fractional calculus, and associated generalized hypergeometric functions, in "Univalent Functions, Fractional Calculus, and Their Applications" (Srivastava, H.M. and Owa, S., Editors), Halsted Press (Ellis Horwood Limited, Chichester), pp. 329-354, John Wiley and Sons, New York, Chichester, Brisbane and Toronto, 1989.

[22] Srivastava, H.M., Mishra, A.K., Gochhayat, P., Certain subclasses of analytic and biunivalent functions, Appl. Math. Lett., 23(2010), 1188-1192. 
[23] Srivastava, H.M., Bansal, D., Close-to-convexity of a certain family of q-Mittag-Leffler functions, J. Nonlinear Var. Anal., 1(2017), 61-69.

[24] Srivastava, H.M., Bulut, S., Çağlar, M., Yağmur, N., Coefficient estimates for a general subclass of analytic and bi-univalent functions, Filomat, 27(2013), 831-842.

[25] Srivastava, H.M., Gaboury, S., Ghanim, F., Coefficient estimates for some general subclasses of analytic and bi-univalent functions, Afrika Mat., 28(2017), 693-706.

[26] Srivastava, H.M., Sümer Eker, S., Ali, R.M., Coefficient bounds for a certain class of analytic and bi-univalent functions, Filomat, 29(2015), 1839-1845.

[27] Todorov, P.G., On the Faber polynomials of the univalent functions of class $\Sigma$, J. Math. Anal. Appl., 162(1991), 268-276.

[28] Xu, Q.-H., Gui, Y.-C., Srivastava, H.M., Coefficient estimates for a certain subclass of analytic and bi-univalent functions, Appl. Math. Lett., 25(2012), 990-994.

[29] Xu, Q.-H., Xiao, H.-G., Srivastava, H.M., A certain general subclass of analytic and biunivalent functions and associated coefficient estimate problems, Appl. Math. Comput., 218(2012), 11461-11465.

[30] Uçar, H.E.Ö., Coefficient inequality for q-starlike functions, Appl. Math. Comput., 276(2016), 122-126.

Hari Mohan Srivastava

Department of Mathematics and Statistics

University of Victoria

Victoria, British Columbia V8W 3R4, Canada

and

Department of Medical Research

China Medical University Hospital

China Medical University, Taichung 40402

Taiwan, Republic of China

e-mail: harimsri@math.uvic.ca

Shahid Khan

Department of Mathematics

Riphah International University

Islamabad, Pakistan

e-mail: shahidmath761@gmail.com

Qazi Zahoor Ahmad

Department of Mathematics

Abbottabad University of Science and Technology

Abbottabad, Pakistan

e-mail: zahoorqazi5@gmail.com

Nazar Khan

Department of Mathematics

Abbottabad University of Science and Technology

Abbottabad, Pakistan

e-mail: nazarmaths@gmail.com 
Saqib Hussain

Department of Mathematics

COMSATS Institute of Information Technology

Abbottabad, Pakistan

e-mail: saqibmath@yahoo.com 